\title{
Synthetic Studies on Cyathins: Enantioselective Total Synthesis of (+)-Allocyathin $B_{2}$
}

\author{
Masashi Takano, Akinori Umino, and Masahisa Nakada* \\ Department of Chemistry, School of Science and Engineering, Waseda University \\ 3-4-1 Ohkubo, Shinjuku-ku, Tokyo 169-8555, Japan
}

General Information. ${ }^{1} \mathrm{H}$ and ${ }^{13} \mathrm{C}$ NMR spectra were recorded on a JEOL AL-400 spectrometer. ${ }^{1} \mathrm{H}$ and ${ }^{13} \mathrm{C}$ chemical shifts are reported in ppm downfield from tetramethylsilane (TMS, $\delta$ scale) with the solvent resonances as internal standards. The following abbreviations were used to explain the multiplicities: s, singlet; $d$, doublet; $t$, triplet; q, quartet; sept, septet; m, multiplet; band, several overlapping signals; br, broad. IR spectra were recorded on a JASCO FT/IR-8300. Melting points $(\mathrm{mp})$ are uncorrected, recorded on a Yamato capillary melting point apparatus. Optical rotations were measured using a $2 \mathrm{ml}$ cell with a $1 \mathrm{dm}$ path length on a JASCO DIP-1000. Mass spectra and elemental analyses were provided at the Materials Characterization Central Laboratory, Waseda University. All reactions were carried out under an argon atmosphere with dry, freshly distilled solvents under anhydrous conditions, unless otherwise noted. All reactions were monitored by thin-layer chromatography carried out on $0.25 \mathrm{~mm}$ E. Merck silica gel plates (60F-254) using UV light as visualizing agent and phosphomolybdic acid and heat as developing agents. E. Merck silica gel (60, particle size $0.040-0.063 \mathrm{~mm}$ ) was used for flash column chromatography. Preparative thin-layer chromatography (PTLC) separations were carried out on self-made $0.3 \mathrm{~mm}$ E. Merck silica gel plates (60F-254).

Materials. THF was distilled from sodium/benzophenone ketyl, and methylene chloride $\left(\mathrm{CH}_{2} \mathrm{Cl}_{2}\right)$, and acetonitrile from calcium hydride. Toluene was distilled from sodium. DMF was distilled from $\mathrm{CaH}_{2}$ under reduced pressure.

\section{(R)-2-(Mesitylsulfonyl)-3-methyl-3-((phenylthio)methyl)cyclopentanone \\ $\mathrm{PhS}$<smiles>CCC1(C)CCC(=O)C1[Sn](C)(C)C</smiles>

a mixture of diastereomers $(1: 6)$

$\mathrm{R}_{\mathrm{f}}=0.33$ (hexane/ethyl acetate $=4 / 1$ ); a white solid; ${ }^{1} \mathrm{H}$ NMR (400 MHz, $\left.\mathrm{CDCl}_{3}\right) \delta$ major: $7.38(2 \mathrm{H}, \mathrm{d}$, $J=8.2 \mathrm{~Hz}), 7.29$ (2H, dd, $J=8.2,7.3 \mathrm{~Hz}), 7.20(1 \mathrm{H}, \mathrm{t}, J=7.3 \mathrm{~Hz}), 6.96(2 \mathrm{H}, \mathrm{s}), 4.18(1 \mathrm{H}, \mathrm{s}), 3.60$ $(1 \mathrm{H}, \mathrm{d}, J=13.1 \mathrm{~Hz}), 3.34(1 \mathrm{H}, \mathrm{d}, J=13.1 \mathrm{~Hz}), 2.65(6 \mathrm{H}, \mathrm{s}), 2.68-2.45(2 \mathrm{H}, \mathrm{m}), 2.30(3 \mathrm{H}, \mathrm{s})$, 2.04-1.89 (2H, m), $1.66(3 \mathrm{H}, \mathrm{s})$; minor: $7.45(2 \mathrm{H}, \mathrm{d}, J=8.2 \mathrm{~Hz}), 7.29(2 \mathrm{H}, \mathrm{dd}, J=8.2,7.3 \mathrm{~Hz}), 7.20$ 
$(1 \mathrm{H}, \mathrm{t}, J=7.3 \mathrm{~Hz}), 6.96(2 \mathrm{H}, \mathrm{s}), 4.18(1 \mathrm{H}, \mathrm{s}), 3.82(1 \mathrm{H}, \mathrm{d}, J=13.3 \mathrm{~Hz}), 3.58(1 \mathrm{H}, \mathrm{d}, J=13.3 \mathrm{~Hz}), 2.63$ $(6 \mathrm{H}, \mathrm{s}), 2.68-2.45(2 \mathrm{H}, \mathrm{m}), 2.30(3 \mathrm{H}, \mathrm{s}), 2.04-1.89(2 \mathrm{H}, \mathrm{m}), 1.66(3 \mathrm{H}, \mathrm{s})$; IR $(\mathrm{KBr}) v_{\max } 1752,1306$, $1138,740,693 \mathrm{~cm}^{-1}$; FAB-MASS $[\mathrm{M}+\mathrm{H}]^{+}$calculated for $\mathrm{C}_{22} \mathrm{H}_{27} \mathrm{O}_{3} \mathrm{~S}_{2}: 403.1402$, found: 403.1382 .

\section{(S)-3-Methyl-3-((phenylthio)methyl)cyclopentanone (8)}

$\mathrm{PhS}$

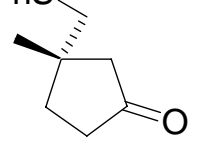

$\mathrm{R}_{\mathrm{f}}=0.35$ (hexane/ethyl acetate $\left.=4 / 1\right)$; a colorless oil; ${ }^{1} \mathrm{H}$ NMR $\left(400 \mathrm{MHz}, \mathrm{CDCl}_{3}\right) \delta 7.36(2 \mathrm{H}, \mathrm{d}, J=$ $7.9 \mathrm{~Hz}), 7.27(2 \mathrm{H}, \mathrm{dd}, J=7.9,7.3 \mathrm{~Hz}), 7.18(1 \mathrm{H}, \mathrm{t}, J=7.3 \mathrm{~Hz}), 3.07(2 \mathrm{H}, \mathrm{s}), 2.32(1 \mathrm{H}, \mathrm{d}, J=18.1 \mathrm{~Hz})$, 2.33-2.29 (2H, m), $2.08(1 \mathrm{H}, \mathrm{d}, J=18.1 \mathrm{~Hz}), 2.03-1.98(1 \mathrm{H}, \mathrm{m}), 1.85-1.78(1 \mathrm{H}, \mathrm{m}), 1.20(3 \mathrm{H}, \mathrm{s}) ;{ }^{13} \mathrm{C}$ NMR (100 MHz, $\left.\mathrm{CDCl}_{3}\right) \delta 218.2,137.1,129.5,128.9,126.2,51.2,46.2,41.1,36.8,34.4$, 25.6; IR (neat) $v_{\max } 1742,740,692 \mathrm{~cm}^{-1}$; FAB-MASS $[\mathrm{M}+\mathrm{H}]^{+}$calculated for $\mathrm{C}_{13} \mathrm{H}_{17} \mathrm{OS}: 221.1000$, found: $221.1003 ;[\alpha]_{\mathrm{D}}^{23}-9.2\left(\mathrm{c} 1.87, \mathrm{CHCl}_{3}\right)$.

\section{(S)-7-Methyl-7-phenylsulfanylmethyl-1,4-dioxa-spiro[4.4]nonane}<smiles>C[C@]1(CS)CCC2(C1)OCCO2</smiles>

$\mathrm{R}_{\mathrm{f}}=0.45$ (hexane/ethyl acetate $\left.=4 / 1\right)$; a colorless oil; ${ }^{1} \mathrm{H}$ NMR $\left(400 \mathrm{MHz}, \mathrm{CDCl}_{3}\right) \delta 7.35(2 \mathrm{H}, \mathrm{dt}, J=$ 7.8, $1.2 \mathrm{~Hz}), 7.25(2 \mathrm{H}, \mathrm{dd}, J=7.8,7.4 \mathrm{~Hz}), 7.14(1 \mathrm{H}, \mathrm{tt}, J=7.4,1.2 \mathrm{~Hz}), 3.89-3.82(4 \mathrm{H}, \mathrm{m}), 3.05(2 \mathrm{H}$, s), $1.99-1.87(2 \mathrm{H}, \mathrm{m}), 1.97(1 \mathrm{H}, \mathrm{d}, J=13.9 \mathrm{~Hz}), 1.81-1.74(1 \mathrm{H}, \mathrm{m}), 1.73(1 \mathrm{H}, \mathrm{d}, J=13.9 \mathrm{~Hz})$, 1.61-1.55 (1H, m), $1.19(3 \mathrm{H}, \mathrm{s}) ;{ }^{13} \mathrm{C}$ NMR $\left(100 \mathrm{MHz}, \mathrm{CDCl}_{3}\right) \delta 137.9,129.0,128.7,125.5,117.7$, 64.2, 64.0, 48.7, 46.8, 41.7, 36.7, 35.7, 26.7; IR (neat) $v_{\max } 1338,1112,1026,738,690 \mathrm{~cm}^{-1}$; FAB-MASS $[\mathrm{M}+\mathrm{H}]^{+}$calculated for $\mathrm{C}_{15} \mathrm{H}_{21} \mathrm{O}_{2} \mathrm{~S}: 265.1262$, found: $265.1260 ;[\alpha]_{\mathrm{D}}{ }^{33}+21.5$ (c 1.49 , $\left.\mathrm{CHCl}_{3}\right)$.

\section{(S)-7-Benzenesulfinylmethyl-7-methyl-1,4-dioxa-spiro[4.4]nonane}

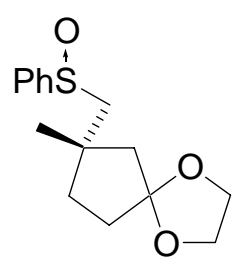

a mixture of diastereomers $(1: 1.1)$

$\mathrm{R}_{\mathrm{f}}=0.09$ (hexane/ethyl acetate $=4 / 1$ ); a pale yellow oil; ${ }^{1} \mathrm{H}$ NMR $\left(400 \mathrm{MHz}, \mathrm{CDCl}_{3}\right) \delta$ major: 
7.65-7.62 (2H, m), 7.54-7.45 (3H, m), 3.92-3.87 (4H, m), $2.93(1 \mathrm{H}, \mathrm{d}, J=12.3 \mathrm{~Hz}), 2.78(1 \mathrm{H}, \mathrm{d}, J=$ $12.3 \mathrm{~Hz}), 2.14-1.69(6 \mathrm{H}, \mathrm{m}), 1.42(3 \mathrm{H}, \mathrm{s})$; minor 7.65-7.62 $(2 \mathrm{H}, \mathrm{m}), 7.54-7.45(3 \mathrm{H}, \mathrm{m}), 3.92-3.87$ $(4 \mathrm{H}, \mathrm{m}), 2.96(1 \mathrm{H}, \mathrm{d}, J=12.3 \mathrm{~Hz}), 2.70(1 \mathrm{H}, \mathrm{d}, J=12.3 \mathrm{~Hz}), 2.14-1.69(6 \mathrm{H}, \mathrm{m}), 1.42(3 \mathrm{H}, \mathrm{s}) ; \mathrm{IR}$ (neat) $v_{\max } 1340,1152,1090,1038,754,692 \mathrm{~cm}^{-1}$; FAB-MASS $[\mathrm{M}+\mathrm{H}]^{+}$calculated for $\mathrm{C}_{15} \mathrm{H}_{21} \mathrm{O}_{3} \mathrm{~S}$ : 281.1211, found: 281.1208 .

(S)-7-Methyl-1,4-dioxa-spiro[4.4]nonane-7-carbaldehyde (5)<smiles>C[C@]1(C=O)CCC2(C1)OCCO2</smiles>

$\mathrm{R}_{\mathrm{f}}=0.45$ (hexane/ethyl acetate $\left.=2 / 1\right)$; a pale yellow oil; ${ }^{1} \mathrm{H}$ NMR $\left(400 \mathrm{MHz}, \mathrm{CDCl}_{3}\right) \delta 9.50(1 \mathrm{H}, \mathrm{s})$, 3.94-3.87 (4H, m), $2.31(1 \mathrm{H}, \mathrm{d}, J=14.2 \mathrm{~Hz}), 2.17-2.08(1 \mathrm{H}, \mathrm{m}), 1.95(1 \mathrm{H}, \mathrm{ddd}, J=13.4,6.8,6.8 \mathrm{~Hz})$, $1.83(1 \mathrm{H}, \mathrm{ddd}, J=13.4,6.8,6.8 \mathrm{~Hz}), 1.67(1 \mathrm{H}, \mathrm{d}, J=14.2 \mathrm{~Hz}), 1.58-1.51(1 \mathrm{H}, \mathrm{m}), 1.2(3 \mathrm{H}, \mathrm{s}) ;{ }^{13} \mathrm{C}$ NMR $\left(100 \mathrm{MHz}, \mathrm{CDCl}_{3}\right) \delta 203.2,117.1,64.2,64.1,51.1,43.3,35.1,31.4,21.3$; IR (neat) $v_{\max }$ 1728, 1338, 1106, $1022 \mathrm{~cm}^{-1}$; FAB-MASS $[\mathrm{M}+\mathrm{H}]^{+}$calculated for $\mathrm{C}_{9} \mathrm{H}_{15} \mathrm{O}_{3}: 171.1021$, found: $171.1023 ;[\alpha]_{\mathrm{D}}^{22}+9.97\left(\mathrm{c} 0.93, \mathrm{CHCl}_{3}\right)$.

$(2 R, 4 a R, 5 S, 8 \mathrm{a} S)-H e x a h y d r o-2-(4-m e t h o x y p h e n y l)-4 a-m e t h y l-4 H-b e n z o[d][1,3]$ dioxin-5-ol (10)<smiles>O[C@H]1OC[C@@H]2[C@H](O)CCC[C@H]2O[C@@H]1P</smiles>

$\mathrm{R}_{\mathrm{f}}=0.24$ (hexane/ethyl acetate $=2 / 1$ ); a white solid mp 91.8-92.3 ${ }^{\circ} \mathrm{C} ;{ }^{1} \mathrm{H}$ NMR (400 MHz, $\left.\mathrm{CDCl}_{3}\right) \delta$ $7.42(2 \mathrm{H}, \mathrm{d}, J=8.8 \mathrm{~Hz}), 6.89(2 \mathrm{H}, \mathrm{d}, J=8.8 \mathrm{~Hz}), 5.47(1 \mathrm{H}, \mathrm{s}), 4.47-4.43(1 \mathrm{H}, \mathrm{m}), 4.26(1 \mathrm{H}, \mathrm{d}, J=11.5$ $\mathrm{Hz}), 3.80(3 \mathrm{H}, \mathrm{s}), 3.80(1 \mathrm{H}, \mathrm{s}), 3.40(1 \mathrm{H}, \mathrm{d}, J=11.5 \mathrm{~Hz}), 1.83-1.68(4 \mathrm{H}, \mathrm{m}), 1.58-1.50(2 \mathrm{H}, \mathrm{m}), 0.79$ $(3 \mathrm{H}, \mathrm{s}) ;{ }^{13} \mathrm{C} \mathrm{NMR}\left(100 \mathrm{MHz}, \mathrm{CDCl}_{3}\right) \delta 159.9,131.3,127.4,113.6,101.7,81.9,72.9,67.7,55.3,37.4$, 30.4, 26.9, 20.0, 13.3; IR (KBr) $v_{\max } 3375,1615,1588,1518,1250,1226,1052,825,783 \mathrm{~cm}^{-1}$; FAB-MASS $[\mathrm{M}+\mathrm{H}]^{+}$calculated for $\mathrm{C}_{16} \mathrm{H}_{23} \mathrm{O}_{4}: 279.1596$, found: $279.1594 ;[\alpha]_{\mathrm{D}}{ }^{21}+52.8$ (c 1.04, $\left.\mathrm{CHCl}_{3}\right)$.

((2R,4aS,5S,8aS)-Hexahydro-2-(4-methoxyphenyl)-4a-methyl-4H-benzo[d][1,3]dioxin-5-yloxy)( tert-butyl)dimethylsilane 


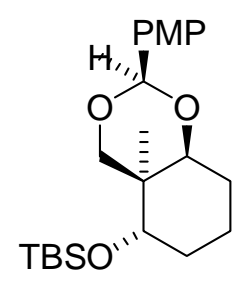

$\mathrm{R}_{\mathrm{f}}=0.70$ (hexane/ethyl acetate $=2 / 1$ ); a white solid; mp 95.2-96.2 ${ }^{\circ} \mathrm{C} ;{ }^{1} \mathrm{H} \mathrm{NMR}\left(400 \mathrm{MHz}, \mathrm{CDCl}_{3}\right) \delta$ $7.41(2 \mathrm{H}, \mathrm{d}, J=8.7 \mathrm{~Hz}), 6.89(2 \mathrm{H}, J=8.7 \mathrm{~Hz}), 4.41(1 \mathrm{H}, \mathrm{dd}, J=11.2,4.4 \mathrm{~Hz}), 4.15(1 \mathrm{H}, \mathrm{d}, J=11.2$ $\mathrm{Hz}), 3.80(3 \mathrm{H}, \mathrm{s}), 3.80(1 \mathrm{H}, \mathrm{s}), 3.31(1 \mathrm{H}, \mathrm{d}, J=11.2 \mathrm{~Hz}), 1.79-1.66(4 \mathrm{H}, \mathrm{m}), 1.58-1.48(2 \mathrm{H}, \mathrm{m}), 0.89$ $(9 \mathrm{H}, \mathrm{s}), 0.75(3 \mathrm{H}, \mathrm{s}), 0.10(3 \mathrm{H}, \mathrm{s}), 0.09(3 \mathrm{H}, \mathrm{s}) ;{ }^{13} \mathrm{C} \mathrm{NMR}\left(100 \mathrm{MHz}, \mathrm{CDCl}_{3}\right) \delta 159.9,131.6,127.5$, $113.6,101.8,82.1,73.1,68.3,55.3,38.0,30.6,26.9,26.0,19.9,18.1,13.7,-3.9,-4.9$; IR (KBr) $v_{\max }$ $1616,1519,1249,1224,1033,774 \mathrm{~cm}^{-1}$; FAB-MASS $[\mathrm{M}+\mathrm{H}]^{+}$calculated for $\mathrm{C}_{22} \mathrm{H}_{37} \mathrm{O}_{4} \mathrm{Si}: 393.2461$, found: $393.2460 ;[\alpha]_{\mathrm{D}}^{22}+48.9\left(\right.$ c $\left.1.01, \mathrm{CHCl}_{3}\right)$.

[(1S,2S,6S)-2-(tert-Butyl-dimethyl-silanyloxy)-6-(4-methoxy-benzyloxy)-1-methyl-cyclohexyl]methanol (11)<smiles>CO[C@H]1CCC[C@@H](O[AsH3])[C@@H]1CO</smiles>

$\mathrm{R}_{\mathrm{f}}=0.62$ (hexane/ethyl acetate $\left.=2 / 1\right)$; a colorless oil; ${ }^{1} \mathrm{H}$ NMR $\left(400 \mathrm{MHz}, \mathrm{CDCl}_{3}\right) \delta 7.25(2 \mathrm{H}, \mathrm{d}, J=$ $8.4 \mathrm{~Hz}), 6.88(2 \mathrm{H}, \mathrm{d}, J=8.4 \mathrm{~Hz}), 4.53(1 \mathrm{H}, \mathrm{d}, J=11.1 \mathrm{~Hz}), 4.25(1 \mathrm{H}, \mathrm{d}, J=11.1 \mathrm{~Hz}), 4.02(1 \mathrm{H}, \mathrm{dd}, J=$ 9.5, $3.7 \mathrm{~Hz}), 3.79(3 \mathrm{H}, \mathrm{s}), 3.68(1 \mathrm{H}, \mathrm{dd}, J=10.4,8.1 \mathrm{~Hz}), 3.57(1 \mathrm{H}, \mathrm{s}), 3.39(1 \mathrm{H}, \mathrm{d}, J=10.4 \mathrm{~Hz}), 3.20$ (1H, br), 1.78-1.73 (1H, m), 1.64-1.43 (5H, m), $0.88(9 \mathrm{H}, \mathrm{s}), 0.84(3 \mathrm{H}, \mathrm{s}), 0.07(3 \mathrm{H}, \mathrm{s}), 0.04(3 \mathrm{H}, \mathrm{s})$; ${ }^{13} \mathrm{C}$ NMR $\left(100 \mathrm{MHz}, \mathrm{CDCl}_{3}\right) \delta 159.1,130.0,129.3,113.8,83.8,70.4,69.6,68.9,55.2,44.0,30.4$, 25.8, 24.1, 18.8, 18.0, 15.5, -4.1, -5.0; IR (neat) $v_{\max } 3516,1614,1516,1252,1038,776 \mathrm{~cm}^{-1}$; FAB-MASS $[\mathrm{M}+\mathrm{H}]^{+}$calculated for $\mathrm{C}_{22} \mathrm{H}_{39} \mathrm{O}_{4} \mathrm{Si}: 395.2618$, found: $395.2615 ;[\alpha]_{\mathrm{D}}{ }^{28}+68.6$ (c 1.04 , $\left.\mathrm{CHCl}_{3}\right)$.

((1S,2S,3S)-3-(4-Methoxybenzyloxy)-2-(iodomethyl)-2-methylcyclohexyloxy)(tert-butyl)dimethy Isilane (6)<smiles>CO[C@H]1CCC[C@@H](O[AsH3])[C@@H]1CI</smiles>

$\mathrm{R}_{\mathrm{f}}=0.65$ (hexane/ethyl acetate $=4 / 1$ ); a white solid mp 53.3-54.1 ${ }^{\circ} \mathrm{C} ;{ }^{1} \mathrm{H}$ NMR $\left(400 \mathrm{MHz}, \mathrm{CDCl}_{3}\right) \delta$ 
$7.32(2 \mathrm{H}, \mathrm{d}, J=8,7 \mathrm{~Hz}), 6.89(2 \mathrm{H}, \mathrm{d}, J=8.7 \mathrm{~Hz}), 4.47(1 \mathrm{H}, \mathrm{d}, J=10.5 \mathrm{~Hz}), 4.36(1 \mathrm{H}, \mathrm{d}, J=10.5 \mathrm{~Hz})$, 3.85-3.82 (1H, m), $3.82(3 \mathrm{H}, \mathrm{s}), 3.66(1 \mathrm{H}, \mathrm{d}, J=9.0 \mathrm{~Hz}), 3.52-3.51(1 \mathrm{H}, \mathrm{m}), 3.45(1 \mathrm{H}, \mathrm{d}, J=9.0 \mathrm{~Hz})$, $1.78-1.70(1 \mathrm{H}, \mathrm{m}), 1.62-1.41(5 \mathrm{H}, \mathrm{m}), 0.98(3 \mathrm{H}, \mathrm{s}), 0.87(9 \mathrm{H}, \mathrm{s}), 0.02(3 \mathrm{H}, \mathrm{s}), 0.01(3 \mathrm{H}, \mathrm{s}) ;{ }^{13} \mathrm{C}$ $\operatorname{NMR}\left(100 \mathrm{MHz}, \mathrm{CDCl}_{3}\right) \delta 159.0,131.1,129.3,113.6,80.7,72.5,71.1,55.3,43.5,31.4,25.9,23.7$, $22.1,19.0,18.0,-3.8,-4.8$; IR (KBr) $v_{\max } 1613,1515,1251,1093,771,560 \mathrm{~cm}^{-1}$; FAB-MASS $[\mathrm{M}-\mathrm{H}]^{+}$calculated for $\mathrm{C}_{22} \mathrm{H}_{36} \mathrm{IO}_{3} \mathrm{Si}: 503.1478$, found: 503.1484; $[\alpha]_{\mathrm{D}}{ }^{22}+76.6$ (c 1.05, $\mathrm{CHCl}_{3}$ ).

\section{2-[(1S,2S,6S)-2-(tert-Butyl-dimethyl-silanyloxy)-6-(4-methoxy-benzyloxy)-1-methyl-cyclohexyl]} -1-[(7S)-7-methyl-1,4-dioxa-spiro[4.4]non-7-yl]-ethanol (12)

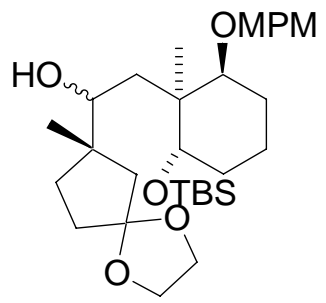

a mixture of diastereomers $(2: 1)$

$\mathrm{R}_{\mathrm{f}}=0.21$ (hexane/ethyl acetate $\left.=4 / 1\right)$; a colorless oil; ${ }^{1} \mathrm{H}$ NMR $\left(400 \mathrm{MHz}, \mathrm{CDCl}_{3}\right) \delta$ major: $7.23(2 \mathrm{H}$, d, $J=8.5 \mathrm{~Hz}), 6.86(2 \mathrm{H}, \mathrm{d}, J=8.5 \mathrm{~Hz}), 4.57(1 \mathrm{H}, \mathrm{d}, J=11.4 \mathrm{~Hz}), 4.41$ (1H, br), 4.33 (1H, d, $J=11.4$ $\mathrm{Hz}), 3.93-3.85(4 \mathrm{H}, \mathrm{m}), 3.81(3 \mathrm{H}, \mathrm{s}), 3.61-3.56(1 \mathrm{H}, \mathrm{m}), 3.50-3.48(1 \mathrm{H}, \mathrm{m}), 3.43-3.42(1 \mathrm{H}, \mathrm{m})$, 1.98-1.37 (14H, m), $1.03(3 \mathrm{H}, \mathrm{s}), 0.96(3 \mathrm{H}, \mathrm{s}), 0.86(9 \mathrm{H}, \mathrm{s}), 0.01(6 \mathrm{H}, \mathrm{s})$; minor: $7.23(2 \mathrm{H}, \mathrm{d}, J=8.5$ $\mathrm{Hz}), 6.86(2 \mathrm{H}, \mathrm{d}, J=8.5 \mathrm{~Hz}), 4.52(1 \mathrm{H}, \mathrm{d}, J=11.2 \mathrm{~Hz}), 4.26(1 \mathrm{H}, \mathrm{d}, J=11.2 \mathrm{~Hz}), 3.93-3.85(5 \mathrm{H}, \mathrm{m})$, $3.81(3 \mathrm{H}, \mathrm{s}), 3.61-3.56(1 \mathrm{H}, \mathrm{m}), 3.50-3.48(1 \mathrm{H}, \mathrm{m}), 1.98-1.37(14 \mathrm{H}, \mathrm{m}), 1.01(3 \mathrm{H}, \mathrm{s}), 1.00(3 \mathrm{H}, \mathrm{s})$, $0.88(9 \mathrm{H}, \mathrm{s}), 0.04(3 \mathrm{H}, \mathrm{s}), 0.03(3 \mathrm{H}, \mathrm{s})$; IR (neat) $v_{\max } 3432,1516,1336,1252,1034,776 \mathrm{~cm}^{-1}$; FAB-MASS [M+H] $]^{+}$calculated for $\mathrm{C}_{31} \mathrm{H}_{53} \mathrm{O}_{6} \mathrm{Si}: 549.3611$, found: 549.3633 .

Dithiocarbonic $\quad$ Acid $\quad O$-[2-[(1S,2S,6S)-2-(tert-Butyl-dimethyl-silanyloxy)-6-(4-methoxybenzyloxy)-1-methyl-cyclohexyl]-1-((7S)-7-methyl-1,4-dioxa-spiro[4.4]non-7-yl)-ethyl] Ester $S$-Methyl Ester (13)

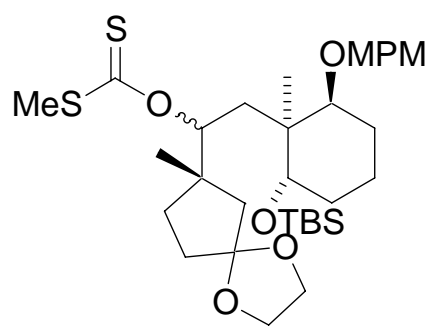

a mixture of diastereomers $(1: 1.5)$

$\mathrm{R}_{\mathrm{f}}=0.48$ (hexane/ethyl acetate $\left.=4 / 1\right)$; an yellow oil; ${ }^{1} \mathrm{H}$ NMR $\left(400 \mathrm{MHz}, \mathrm{CDCl}_{3}\right) \delta$ major: $7.33(2 \mathrm{H}$, d, $J=8.5 \mathrm{~Hz}), 6.89(2 \mathrm{H}, \mathrm{d}, J=8.5 \mathrm{~Hz}), 6.05(1 \mathrm{H}, \mathrm{d}, J=9.8 \mathrm{~Hz}), 4.41(1 \mathrm{H}, \mathrm{d}, J=11.0 \mathrm{~Hz}), 4.27(1 \mathrm{H}, \mathrm{d}$, 
$J=11.0 \mathrm{~Hz}), 3.94-3.82(5 \mathrm{H}, \mathrm{m}), 3.83(3 \mathrm{H}, \mathrm{s}), 3.49-3.47(1 \mathrm{H}, \mathrm{m}), 2.48(3 \mathrm{H}, \mathrm{s}), 2.31-1.39(14 \mathrm{H}, \mathrm{m})$, $1.18(3 \mathrm{H}, \mathrm{s}), 1.02(3 \mathrm{H}, \mathrm{s}), 0.88(9 \mathrm{H}, \mathrm{s}), 0.03(6 \mathrm{H}, \mathrm{s})$; minor: $7.27(2 \mathrm{H}, \mathrm{d}, J=8.5 \mathrm{~Hz}), 6.88(2 \mathrm{H}, \mathrm{d}, J=$ $8.5 \mathrm{~Hz}), 6.05(1 \mathrm{H}, \mathrm{d}, J=9.8 \mathrm{~Hz}), 4.52(1 \mathrm{H}, \mathrm{d}, J=11.0 \mathrm{~Hz}), 4.33(1 \mathrm{H}, \mathrm{d}, J=11.0 \mathrm{~Hz}), 3.94-3.82(4 \mathrm{H}$, m), $3.82(3 \mathrm{H}, \mathrm{s}), 3.72-3.70(2 \mathrm{H}, \mathrm{m}), 2.55(3 \mathrm{H}, \mathrm{s}), 2.31-1.39(14 \mathrm{H}, \mathrm{m}), 1.11(3 \mathrm{H}, \mathrm{s}), 0.94(3 \mathrm{H}, \mathrm{s}), 0.88$ $(9 \mathrm{H}, \mathrm{s}), 0.03(3 \mathrm{H}, \mathrm{s}), 0.01(3 \mathrm{H}, \mathrm{s})$; IR (neat) $v_{\max } 1616,1516,1338,1250,1042,776 \mathrm{~cm}^{-1}$; FAB-MASS [M-H] $]^{+}$calculated for $\mathrm{C}_{33} \mathrm{H}_{55} \mathrm{O}_{6} \mathrm{~S}_{2} \mathrm{Si}$ : 637.3053, found: 637.3067 .

(+)-tert-Butyl-((1S,2S,3S)-3-(4-methoxy-benzyloxy)-2-methyl-2-((S)-2-(7-methyl-1,4-dioxa-spiro [4.4]non-7-yl)-ethyl)-cyclohexyloxy)-dimethyl-silane (14)

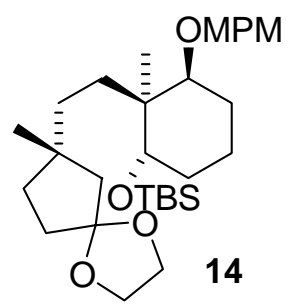

$\mathrm{R}_{\mathrm{f}}=0.31$ (hexane/ethyl acetate=10/1); colorless oil; ${ }^{1} \mathrm{H}$ NMR (400 MHz, $\left.\mathrm{CDCl}_{3}\right) \delta 7.25(2 \mathrm{H}, \mathrm{d}, J=8.5$ $\mathrm{Hz}), 6.86(2 \mathrm{H}, \mathrm{d}, J=8.5 \mathrm{~Hz}), 4.48(1 \mathrm{H}, \mathrm{d}, J=11.2 \mathrm{~Hz}), 4.23(1 \mathrm{H}, \mathrm{d}, J=11.2 \mathrm{~Hz}), 3.89-3.81(4 \mathrm{H}, \mathrm{m})$, $3.80(3 \mathrm{H}, \mathrm{s}), 3.69(1 \mathrm{H}, \mathrm{dd}, J=8.8,3.6 \mathrm{~Hz}), 3.40(1 \mathrm{H}, \mathrm{dd}, J=5.6,1.7 \mathrm{~Hz}), 1.93-1.80(2 \mathrm{H}, \mathrm{m}), 1.73-1.40$ $(11 \mathrm{H}, \mathrm{m}), 1.38(1 \mathrm{H}, \mathrm{ddd}, J=12.7,12.5,3.6 \mathrm{~Hz}), 1.28(1 \mathrm{H}, \mathrm{ddd}, J=12.7,12.5,4.6 \mathrm{~Hz}), 1.18(1 \mathrm{H}, \mathrm{ddd}$, $J=12.7,12.5,3.6 \mathrm{~Hz}), 0.99(3 \mathrm{H}, \mathrm{s}), 0.87(9 \mathrm{H}, \mathrm{s}), 0.84(3 \mathrm{H}, \mathrm{s}), 0.02(3 \mathrm{H}, \mathrm{s}), 0.01(3 \mathrm{H}, \mathrm{s}) ;{ }^{13} \mathrm{C} \mathrm{NMR}$ $\left(100 \mathrm{MHz}, \mathrm{CDCl}_{3}\right) \delta 158.8,131.4,128.9(2 \mathrm{C}), 118.2,113.5(2 \mathrm{C}), 79.8,74.4,70.3,64.1,63.9,55.3$, 49.4, 42.5, 40.3, 37.4, 35.6, 35.2, 30.2, 29.7, 26.1, 25.9(2C), 23.7, 18.8, 18.1, 17.6, -3.9, -4.7; IR (neat) $v_{\max } 1616,1516,1250,1040,774 \mathrm{~cm}^{-1}$; FAB-MS $[\mathrm{M}+\mathrm{H}]^{+}$calculated for $\mathrm{C}_{31} \mathrm{H}_{52} \mathrm{O}_{5} \mathrm{Si}$ : 533.3662, found : $533.3653 ;[\alpha]_{\mathrm{D}}^{21.9}+59.3\left(\mathrm{c} 1.04, \mathrm{CHCl}_{3}\right)$.

(+)-(S)-3-(2-((1R,2S,6S)-2-(4-Methoxybenzyloxy)-6-hydroxy-1-methylcyclohexyl)ethyl)-3-methy lcyclopentanone<smiles>CO[C@H]1CCCC[C@@]1(C)CC[C@]1(C)CCC(=O)C1</smiles>

$\mathrm{R}_{\mathrm{f}}=0.17$ (hexane/ethyl acetate $=2 / 1$ ); a white solid; mp 79.7-80.7 ${ }^{\circ} \mathrm{C} ;{ }^{1} \mathrm{H}$ NMR $\left(400 \mathrm{MHz}, \mathrm{CDCl}_{3}\right) \delta$ $7.23(2 \mathrm{H}, \mathrm{d}, J=8.5 \mathrm{~Hz}), 6.86(2 \mathrm{H}, \mathrm{d}, J=8.5 \mathrm{~Hz}), 4.51(1 \mathrm{H}, \mathrm{d}, J=11.2 \mathrm{~Hz}), 4.17(1 \mathrm{H}, \mathrm{d}, J=11.2 \mathrm{~Hz})$, $3.80(3 \mathrm{H}, \mathrm{s}), 3.77(1 \mathrm{H}, \mathrm{dd}, J=10.3,4.4 \mathrm{~Hz}), 3.41(1 \mathrm{H}, \mathrm{dd}, J=3.7,1.5 \mathrm{~Hz}), 2.29-2.13(2 \mathrm{H}, \mathrm{m}), 2.04$ $(1 \mathrm{H}, \mathrm{d}, J=18.1 \mathrm{~Hz}), 1.99(1 \mathrm{H}, \mathrm{d}, J=18.1 \mathrm{~Hz}), 1.83-1.51(10 \mathrm{H}, \mathrm{m}), 1.45(1 \mathrm{H}, \mathrm{ddd}, J=12.5,12.0,4.4$ 
$\mathrm{Hz}), 1.33(1 \mathrm{H}, \mathrm{ddd}, J=12.7,11.0,4.6 \mathrm{~Hz}), 1.27(1 \mathrm{H}$, ddd, $J=12.7,12.0,4.4 \mathrm{~Hz}), 1.01(3 \mathrm{H}, \mathrm{s}), 0.88$ $(3 \mathrm{H}, \mathrm{s}) ;{ }^{13} \mathrm{C} \mathrm{NMR}\left(100 \mathrm{MHz}, \mathrm{CDCl}_{3}\right) \delta 220.0,158.9,131.0,128.8(2 \mathrm{C}), 113.6(2 \mathrm{C}), 79.6,73.9,70.0$, 55.3, 52.5, 42.0, 39.4, 36.8, 34.9, 34.3, 30.2, 29.9, 25.1, 23.2, 18.7, 16.3; IR (KBr) $v_{\max } 3309,1742$, $1615,1512,1244,1047,814 \mathrm{~cm}^{-1}$; FAB-MS $[\mathrm{M}]^{+}$calculated for $\mathrm{C}_{23} \mathrm{H}_{34} \mathrm{O}_{4}: 374.2457$, found : $374.2455 ;[\alpha]_{\mathrm{D}}^{24.9}+59.4\left(\mathrm{c} 0.97, \mathrm{CHCl}_{3}\right)$.

(+)-(2S,3S)-3-(4-Methoxybenzyloxy)-2-methyl-2-(2-((S)-1-methyl-3-oxocyclopentyl)ethyl)cycloh exanone (4)

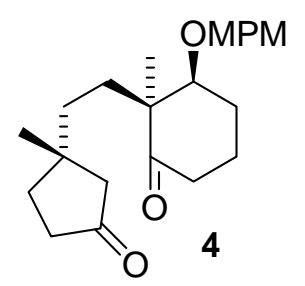

$\mathrm{R}_{\mathrm{f}}=0.36$ (hexane/ethyl acetate $\left.=2 / 1\right)$; a colorless oil; ${ }^{1} \mathrm{H}$ NMR $\left(400 \mathrm{MHz}, \mathrm{CDCl}_{3}\right) \delta 7.22(2 \mathrm{H}, \mathrm{d}, J=8.5$ $\mathrm{Hz}), 6.86(2 \mathrm{H}, \mathrm{d}, J=8.5 \mathrm{~Hz}), 4.56(1 \mathrm{H}, \mathrm{d}, J=11.5 \mathrm{~Hz}), 4.31(1 \mathrm{H}, \mathrm{d}, J=11.5 \mathrm{~Hz}), 3.80(3 \mathrm{H}, \mathrm{s}), 3.40(1 \mathrm{H}$, dd, $J=6.8,3.4 \mathrm{~Hz}), 2.43-2.20(4 \mathrm{H}, \mathrm{m}), 2.02(1 \mathrm{H}, \mathrm{d}, J=10.5 \mathrm{~Hz}), 2.00(1 \mathrm{H}, \mathrm{d}, J=10.5 \mathrm{~Hz}), 2.05-1.91$ $(2 \mathrm{H}, \mathrm{m}), 1.78-1.70(3 \mathrm{H}, \mathrm{m}), 1.65(1 \mathrm{H}, \mathrm{ddd}, J=13.4,12.9,4.4 \mathrm{~Hz}), 1.60-1.52(2 \mathrm{H}, \mathrm{m}), 1.26(1 \mathrm{H}, \mathrm{ddd}$, $J=12.9,12.9,5.1 \mathrm{~Hz}), 1.12(3 \mathrm{H}, \mathrm{s}), 1.06(1 \mathrm{H}, \mathrm{ddd}, J=13.4,13.2,4.4 \mathrm{~Hz}), 1.02(3 \mathrm{H}, \mathrm{s}) ;{ }^{13} \mathrm{C} \mathrm{NMR}$ $\left(100 \mathrm{MHz}, \mathrm{CDCl}_{3}\right) \delta 219.5,213.5,159.0,130.2,129.1(2 \mathrm{C}), 113.6(2 \mathrm{C}), 83.0,70.9,55.2,53.8,52.3$, 39.3, 37.8, 36.7, 34.9, 34.7, 26.9, 24.8, 24.1, 20.4, 19.7; IR (neat) $v_{\max } 1738,1708,1614,1516,1250$, 1036, $822 \mathrm{~cm}^{-1}$; FAB-MS $[\mathrm{M}+\mathrm{H}]^{+}$calculated for $\mathrm{C}_{23} \mathrm{H}_{33} \mathrm{O}_{4}: 373.2379$, found : 373.2380 ; $[\alpha]_{\mathrm{D}} 22.5$ $+24.7\left(\mathrm{c} 0.99, \mathrm{CHCl}_{3}\right)$.

(+)-(3aS,5aS,6S,9aR,9bR)-6-(4-Methoxybenzyloxy)-decahydro-9a-hydroxy-3a,5a-dimethyl-2Hcyclopenta[a]naphthalen-1(9bH)-one (15)

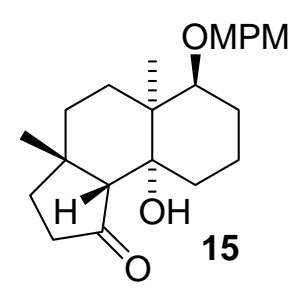

$\mathrm{R}_{\mathrm{f}}=0.28$ (hexane/ethyl acetate $=4 / 1$ ); colorless crystal; mp 134.6-136.8 ${ }^{\circ} \mathrm{C} ;{ }^{1} \mathrm{H}$ NMR (400 MHz, $\left.\mathrm{CDCl}_{3}\right) \delta 7.24(2 \mathrm{H}, \mathrm{d}, J=8.5 \mathrm{~Hz}), 6.85(2 \mathrm{H}, \mathrm{d}, J=8.5 \mathrm{~Hz}), 5.77(1 \mathrm{H}, \mathrm{br}), 4.54(1 \mathrm{H}, \mathrm{d}, J=11.2 \mathrm{~Hz})$, $4.33(1 \mathrm{H}, \mathrm{d}, J=11.2 \mathrm{~Hz}), 3.79$ (3H, s), 3.52 (1H, dd, $J=11.7,4.4 \mathrm{~Hz}), 2.55$ (1H, ddd, $J=19.5,12.0,9.5$ $\mathrm{Hz}), 2.32(1 \mathrm{H}, \mathrm{dd}, J=19.5,8.8 \mathrm{~Hz}), 2.02-1.94(1 \mathrm{H}, \mathrm{m}), 1.90-1.86(1 \mathrm{H}, \mathrm{m}), 1.82-1.48(8 \mathrm{H}, \mathrm{m})$, 1.46-1.35 (2H, m), $1.28(3 \mathrm{H}, \mathrm{s}), 1.26(1 \mathrm{H}, \mathrm{s}), 0.91(3 \mathrm{H}, \mathrm{s}) ;{ }^{13} \mathrm{C} \mathrm{NMR}\left(100 \mathrm{MHz}, \mathrm{CDCl}_{3}\right) \delta 225.5$, 158.7, 131.3, 128.9(2C), 113.5(2C), 81.9, 77.1, 71.6, 62.5, 55.2, 44.2, 40.6, 37.1, 36.3, 35.6, 29.5, 
28.8, 25.6, 23.7, 19.2, 18.7; IR (KBr) $v_{\max } 3405,1707,1615,1514,1253,1078,819 \mathrm{~cm}^{-1}$; FAB-MS $[\mathrm{M}]^{+}$calculated for $\mathrm{C}_{23} \mathrm{H}_{32} \mathrm{O}_{4}: 372.2301$, found : $372.2309 ;[\alpha]_{\mathrm{D}}^{21.1}+138.6\left(\mathrm{c} 0.97, \mathrm{CHCl}_{3}\right)$.

(+)-(3aS,5aR,6S,9bR)-6-(4-Methoxybenzyloxy)-3,3a,4,5,5a,6,7,8-octahydro-3a,5a-dimethyl-2Hcyclopenta[a]naphthalen-1(9bH)-one (16)

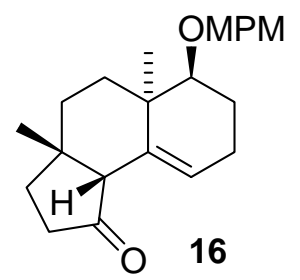

$\mathrm{R}_{\mathrm{f}}=0.36$ (hexane/ethyl acetate $=4 / 1$ ); pale yellow crystal; mp 134.2-135.9 ${ }^{\circ} \mathrm{C} ;{ }^{1} \mathrm{H}$ NMR (400 MHz, $\left.\mathrm{CDCl}_{3}\right) \delta 7.26(2 \mathrm{H}, \mathrm{d}, J=8.5 \mathrm{~Hz}), 6.86(2 \mathrm{H}, \mathrm{d}, J=8.5 \mathrm{~Hz}), 5.42(1 \mathrm{H}, \mathrm{dd}, J=4.2,3.2 \mathrm{~Hz}), 4.58(1 \mathrm{H}, \mathrm{d}$, $J=11.7 \mathrm{~Hz}), 4.38(1 \mathrm{H}, \mathrm{d}, J=11.7 \mathrm{~Hz}), 3.80(3 \mathrm{H}, \mathrm{s}), 3.17(1 \mathrm{H}, \mathrm{dd}, J=4.2,1.7 \mathrm{~Hz}), 2.45(1 \mathrm{H}, \mathrm{ddd}$, $J=19.5,11.7,9.0 \mathrm{~Hz}), 2.36(1 \mathrm{H}, \mathrm{ddd}, J=9.3,1.7,1.7 \mathrm{~Hz}), 2.32-2.20(3 \mathrm{H}, \mathrm{m}), 2.09-2.01(1 \mathrm{H}, \mathrm{m})$, 1.92-1.79 (3H, m), 1.70-1.61 (2H, m), $1.31(1 \mathrm{H}, \mathrm{ddd}, J=13.9,6.3,2.9 \mathrm{~Hz}), 1.15(3 \mathrm{H}, \mathrm{m}), 0.96(1 \mathrm{H}$, ddd, $J=13.7,3.4,3.4 \mathrm{~Hz}), 0.84(3 \mathrm{H}, \mathrm{s}) ;{ }^{13} \mathrm{C} \mathrm{NMR}\left(100 \mathrm{MHz}, \mathrm{CDCl}_{3}\right) \delta 219.5,158.7,133.9,131.3$, 128.8(2C), 127.5, 113.5(2C), 80.6, 70.6, 65.4, 55.2, 40.4, 38.9, 35.6, 35.1, 29.1, 28.4, 26.0, 26.0, 21.6, 21.0; IR (KBr) $v_{\max } 1739,1613,1511,1246,1079,826 \mathrm{~cm}^{-1}$; FAB-MS [M+H] ${ }^{+}$calculated for $\mathrm{C}_{23} \mathrm{H}_{31} \mathrm{O}_{3}: 355.2273$, found : $355.2291 ;[\alpha]_{\mathrm{D}}^{25.8}+126.9\left(\mathrm{c} 1.03, \mathrm{CHCl}_{3}\right)$.

(-)-(3aS,5aR,6S)-6-(4-Methoxybenzyloxy)-2,3,3a,4,5,5a,6,7,8,9-decahydro-3a,5a-dimethyl-cyclo penta[a]naphthalen-1-one (17)

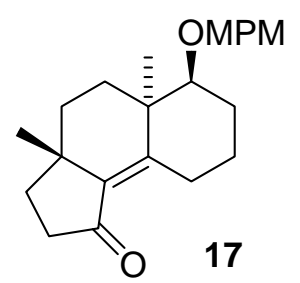

$\mathrm{R}_{\mathrm{f}}=0.45$ (hexane/ethyl acetate $\left.=4 / 1\right)$; a colorless oil; ${ }^{1} \mathrm{H} \mathrm{NMR}\left(400 \mathrm{MHz}, \mathrm{CDCl}_{3}\right) \delta 7.23(2 \mathrm{H}, \mathrm{d}, J=8.6$ Hz), $6.85(2 \mathrm{H}, \mathrm{d}, J=8.6 \mathrm{~Hz}), 4.51(1 \mathrm{H}, \mathrm{d}, J=11.4 \mathrm{~Hz}), 4.27(1 \mathrm{H}, \mathrm{d}, J=11.4 \mathrm{~Hz}), 3.99$ (1H, ddd, $J=13.2$, 1.2, $1.2 \mathrm{~Hz}), 3.80(3 \mathrm{H}, \mathrm{s}), 3.33(1 \mathrm{H}, \mathrm{s}), 2.52(1 \mathrm{H}, \mathrm{ddd}, J=14.1,14.1,2.9 \mathrm{~Hz}), 2.42$ (1H, ddd, $J=18.9$, $12.1,8.6 \mathrm{~Hz}), 2.20(1 \mathrm{H}, \mathrm{dd}, J=18.9,8.6 \mathrm{~Hz}), 1.90-1.71(5 \mathrm{H}, \mathrm{m}), 1.70-1.61(2 \mathrm{H}, \mathrm{m}), 1.52-1.44(2 \mathrm{H}$, m), $1.13(3 \mathrm{H}, \mathrm{s}), 1.07(3 \mathrm{H}, \mathrm{m}), 1.03(1 \mathrm{H}, \mathrm{ddd}, J=13.5,3.3,3.3 \mathrm{~Hz}) ;{ }^{13} \mathrm{C} \mathrm{NMR}\left(100 \mathrm{MHz}, \mathrm{CDCl}_{3}\right) \delta$ 208.4, 158.6, 153.0, 137.6, 130.9, 128.7(2C), 113.4(2C), 83.4, 70.4, 55.3, 42.5, 40.6, 36.9, 35.3, 33.4, 29.2, 25.9, 25.2, 23.6, 22.8, 21.6; IR (neat) $v_{\max } 1704,1626,1516,1250,1072,822 \mathrm{~cm}^{-1}$; FAB-MS $[\mathrm{M}+\mathrm{H}]^{+}$calculated for $\mathrm{C}_{23} \mathrm{H}_{31} \mathrm{O}_{3}: 355.2273$, found : $355.2273 ;[\alpha]_{\mathrm{D}}^{25.0}-139.8$ (c 1.03, $\left.\mathrm{CHCl}_{3}\right)$. 
(+)-(1R,3a $S, 5 \mathrm{a} R, 6 S, 9 \mathrm{~b} R)-6-(4-M e t h o x y b e n z y l o x y)-2,3,3 \mathrm{a}, 4,5,5 \mathrm{a}, 6,7,8,9 \mathrm{~b}-d e c a h y d r o-3 \mathrm{a}, 5 \mathrm{a}-d i m e t$ hyl-1-(prop-1-en-2-yl)-1H-cyclopenta[a]naphthalen-1-ol (18)

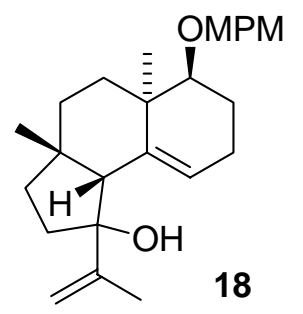

$\mathrm{R}_{\mathrm{f}}=0.38$ (hexane/ethyl acetate $\left.=10 / 1\right)$; a colorless oil; ${ }^{1} \mathrm{H}$ NMR $\left(400 \mathrm{MHz}, \mathrm{CDCl}_{3}\right) \delta 7.26(2 \mathrm{H}, \mathrm{d}$, $J=8.8 \mathrm{~Hz}), 6.86(2 \mathrm{H}, \mathrm{d}, J=8.8 \mathrm{~Hz}), 5.25(1 \mathrm{H}, \mathrm{dd}, J=3.4,3.2 \mathrm{~Hz}), 4.89(1 \mathrm{H}, \mathrm{d}, J=1.0 \mathrm{~Hz}), 4.79(1 \mathrm{H}$, dd, $J=1.5,1.2 \mathrm{~Hz}), 4.57(1 \mathrm{H}, \mathrm{d}, J=12.0 \mathrm{~Hz}), 4.40(1 \mathrm{H}, \mathrm{d}, J=12.0 \mathrm{~Hz}), 3.79(3 \mathrm{H}, \mathrm{s}), 3.18(1 \mathrm{H}, \mathrm{dd}$, $J=2.9,2.4 \mathrm{~Hz}), 2.40(1 \mathrm{H}, \mathrm{s}), 2.28-2.18(1 \mathrm{H}, \mathrm{m}), 2.20(1 \mathrm{H}, \mathrm{ddd}, J=13.9,13.9,3.4 \mathrm{~Hz}), 2.04-1.62(7 \mathrm{H}$, m), $1.78(3 \mathrm{H}, \mathrm{d}, J=0.7 \mathrm{~Hz}), 1.59-1.51(2 \mathrm{H}, \mathrm{m}), 1.32(1 \mathrm{H}, \mathrm{dddd}, J=13.4,3.4,3.4,1.2 \mathrm{~Hz}), 1.10(3 \mathrm{H}$, s), $1.08(3 \mathrm{H}, \mathrm{s}), 0.88(1 \mathrm{H}, \mathrm{ddd}, J=13.4,3.4,3.4 \mathrm{~Hz}) ;{ }^{13} \mathrm{C} \mathrm{NMR}\left(100 \mathrm{MHz}, \mathrm{CDCl}_{3}\right) \delta 158.8,150.7$, 136.1, 131.4, 129.0(2C), 127.5, 113.5(2C), 109.1, 85.6, 81.4, 70.7, 58.6, 55.2, 41.0, 40.8, 37.7, 37.0, 32.2, 29.0, 28.7, 27.8, 21.8, 20.5, 20.0; IR (neat) $v_{\max } 3564,1614,1516,1248,1082,820 \mathrm{~cm}^{-1}$; FAB-MS $[\mathrm{M}+\mathrm{H}]^{+}$calculated for $\mathrm{C}_{26} \mathrm{H}_{37} \mathrm{O}_{3}: 397.2743$, found : $397.2751 ;[\alpha]_{\mathrm{D}}^{23.5}+11.6$ (c 1.02, $\left.\mathrm{CHCl}_{3}\right)$.

(+)-(1S,3aS,5aR,6S,9bR)-2,3,3a,4,5,5a,6,7,8,9b-Decahydro-1-isopropyl-3a,5a-dimethyl-1H-cyclo penta[a]naphthalene-1,6-diol

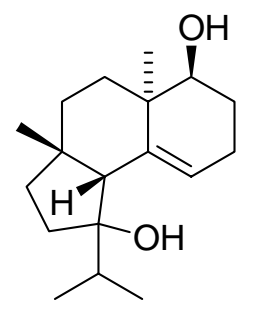

$\mathrm{R}_{\mathrm{f}}=0.29$ (hexane/ethyl acetate $=4 / 1$ ); a white solid; $\mathrm{mp} 45.2-46.8^{\circ} \mathrm{C} ;{ }^{1} \mathrm{H}$ NMR $\left(400 \mathrm{MHz}, \mathrm{CDCl}_{3}\right) \delta$ 5.49 (1H, dd, $J=3.7,2.9 \mathrm{~Hz}), 3.47$ (1H, br), 2.34-2.23 (1H, m), 2.28 (1H, s), 2.17-2.00 (3H, m), 1.95 $(1 \mathrm{H}, \mathrm{ddd}, J=14.2,13.4,3.2 \mathrm{~Hz}), 1.86(1 \mathrm{H}, \mathrm{ddd}, J=12.5,9.5,3.7 \mathrm{~Hz}), 1.78(1 \mathrm{H}, \mathrm{ddd}, J=13.9,7.8,3.7$ $\mathrm{Hz}), 1.70-1.55(4 \mathrm{H}, \mathrm{m}), 1.35$ (1H, ddd, $J=11.2,8.5,8.5 \mathrm{~Hz}), 1.28-1.23(1 \mathrm{H}, \mathrm{m}), 1.13(1 \mathrm{H}, \mathrm{s}), 1.12$ $(3 \mathrm{H}, \mathrm{s}), 1.03(1 \mathrm{H}, \mathrm{ddd}, J=13.4,3.4,3.4 \mathrm{~Hz}), 1.01(3 \mathrm{H}, \mathrm{s}), 0.97(3 \mathrm{H}, \mathrm{d}, J=6.8 \mathrm{~Hz}), 0.93(3 \mathrm{H}, \mathrm{d}, J=6.8$ $\mathrm{Hz}) ;{ }^{13} \mathrm{C} \mathrm{NMR}\left(100 \mathrm{MHz}, \mathrm{CDCl}_{3}\right) \delta 138.0,127.3,85.5,74.7,58.4,41.1,40.0,38.2,37.0,31.6,31.1$, 28.9, 27.7, 27.7, 24.4, 20.9, 18.9, 17.5; IR (KBr) $v_{\max } 3415,1295,1062 \mathrm{~cm}^{-1}$; FAB-MS [M+H] ${ }^{+}$ calculated for $\mathrm{C}_{18} \mathrm{H}_{31} \mathrm{O}_{2}: 279.2324$, found : $279.2321 ;[\alpha]_{\mathrm{D}}^{22.5}+53.4\left(\mathrm{c} 1.01, \mathrm{CHCl}_{3}\right)$. 
(-)-(1S,3aS,5aR,9bR)-2,3,3a,4,5,5a,7,8-Octahydro-1-hydroxy-1-isopropyl-3a,5a-dimethyl-1H-cy clopenta[a]naphthalen-6(9bH)-one (19)

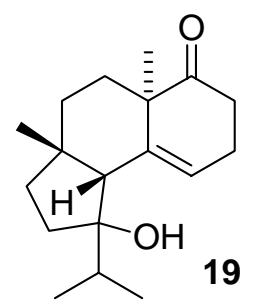

$\mathrm{R}_{\mathrm{f}}=0.38$ (hexane/ethyl acetate $=4 / 1$ ); a white solid; mp 74.8-76.2 ${ }^{\circ} \mathrm{C} ;{ }^{1} \mathrm{H}$ NMR $\left(400 \mathrm{MHz}, \mathrm{CDCl}_{3}\right) \delta$ $5.70(1 \mathrm{H}, \mathrm{dd}, J=6.1,2.9 \mathrm{~Hz}), 2.71(1 \mathrm{H}, \mathrm{ddd}, J=16.4,7.8,4.2 \mathrm{~Hz}), 2.64-2.55(1 \mathrm{H}, \mathrm{m}), 2.43-2.34(1 \mathrm{H}$, m), $2.33(1 \mathrm{H}, \mathrm{s}), 2.31-2.23(1 \mathrm{H}, \mathrm{m}), 1.96-1.88(1 \mathrm{H}, \mathrm{m}), 1.84-1.73(3 \mathrm{H}, \mathrm{m}), 1.67-1.51(3 \mathrm{H}, \mathrm{m})$, 1.38-1.32 (1H, m), $1.31(3 \mathrm{H}, \mathrm{s}), 1.28-1.03(2 \mathrm{H}, \mathrm{m}), 1.00(3 \mathrm{H}, \mathrm{d}, J=6.8 \mathrm{~Hz}), 0.96(3 \mathrm{H}, \mathrm{d}, J=6.8 \mathrm{~Hz})$, $0.94(3 \mathrm{H}, \mathrm{s}) ;{ }^{13} \mathrm{C} \mathrm{NMR}\left(100 \mathrm{MHz}, \mathrm{CDCl}_{3}\right) \delta 215.3,140.6,127.8,86.2,59.0,48.2,40.8,37.9,35.9$, $34.4,32.7,32.3,29.3,27.6,23.4,23.3,18.9,17.5$; IR (KBr) $v_{\max } 3527,1702,1201 \mathrm{~cm}^{-1}$; FAB-MS $[\mathrm{M}+\mathrm{H}]^{+}$calculated for $\mathrm{C}_{18} \mathrm{H}_{29} \mathrm{O}_{2}: 277.2168$, found : 277.2177; $[\alpha]_{\mathrm{D}}^{21.1}-113.7$ (c 1.01, $\left.\mathrm{CHCl}_{3}\right)$.

(+)-(3aR,5aR)-2,3,3a,4,5,5a,7,8-Octahydro-1-isopropyl-3a,5a-dimethylcyclopenta[a]naphthalen -6-one (3)

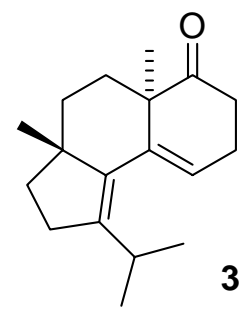

$\mathrm{R}_{\mathrm{f}}=0.36$ (hexane/ethyl acetate $\left.=20 / 1\right)$; a colorless oil; ${ }^{1} \mathrm{H} \mathrm{NMR}\left(400 \mathrm{MHz}, \mathrm{CDCl}_{3}\right) \delta 5.48(1 \mathrm{H}, \mathrm{dd}$, $J=4.2,3.9 \mathrm{~Hz}), 2.87(1 \mathrm{H}$, sept, $J=6.8 \mathrm{~Hz}), 2.71(1 \mathrm{H}, \mathrm{ddd}, J=13.9,7.1,4.9 \mathrm{~Hz}), 2.51-2.40(3 \mathrm{H}, \mathrm{m})$, $2.33(1 \mathrm{H}, \mathrm{dd}, J=9.5,6.1 \mathrm{~Hz}), 2.32(1 \mathrm{H}, \mathrm{dd}, J=9.5,3.7 \mathrm{~Hz}), 1.80-1.51(6 \mathrm{H}, \mathrm{m}), 1.15(3 \mathrm{H}, \mathrm{s}), 1.00(3 \mathrm{H}$, d, $J=6.8 \mathrm{~Hz}), 0.97(3 \mathrm{H}, \mathrm{d}, J=6.8 \mathrm{~Hz}), 0.91(3 \mathrm{H}, \mathrm{s}) ;{ }^{13} \mathrm{C} \mathrm{NMR}\left(100 \mathrm{MHz}, \mathrm{CDCl}_{3}\right) \delta 215.6,142.0$, 139.4, 137.7, 120.9, 48.6, 48.3, 39.4, 36.3, 35.8, 31.4, 28.3, 26.5, 25.5, 23.0, 22.9, 21.6, 21.6; IR (neat) $v_{\max } 1716 \mathrm{~cm}^{-1}$; FAB-MS [M-H] $]^{+}$calculated for $\mathrm{C}_{18} \mathrm{H}_{25} \mathrm{O}: 257.1905$, found : 257.1895; $[\alpha]_{\mathrm{D}}^{21.5}+270.8\left(\mathrm{c} 0.98, \mathrm{CHCl}_{3}\right)$.

(+)-(3aR,5aR)-Methyl

3,3a,4,5,5a,8-hexahydro-6-hydroxy-1-isopropyl-3a,5a-dimethyl-2H-cyclopenta[a]naphthalene-7 -carboxylate 


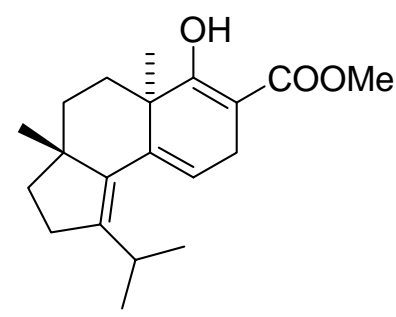

$\mathrm{R}_{\mathrm{f}}=0.51$ (hexane/ethyl acetate $\left.=20 / 1\right)$; a colorless oil; ${ }^{1} \mathrm{H}$ NMR $\left(400 \mathrm{MHz}, \mathrm{CDCl}_{3}\right) \delta 12.38(1 \mathrm{H}, \mathrm{s})$, $5.33(1 \mathrm{H}, \mathrm{dd}, J=3.9,3.4 \mathrm{~Hz}), 3.78(3 \mathrm{H}, \mathrm{s}), 3.00(1 \mathrm{H}, \mathrm{dd}, J=21.5,4.4 \mathrm{~Hz}), 2.93(1 \mathrm{H}, \mathrm{dd}, J=21.5,3.2$ $\mathrm{Hz}), 2.83(1 \mathrm{H}$, sept, $J=6.8 \mathrm{~Hz}), 2.32(1 \mathrm{H}, \mathrm{dd}, J=9.5,6.6 \mathrm{~Hz}), 2.31(1 \mathrm{H}, \mathrm{dd}, J=9.5,3.4 \mathrm{~Hz}), 1.96(1 \mathrm{H}$, ddd, $J=13.4,3.7,3.4 \mathrm{~Hz}), 1.84-1.76(1 \mathrm{H}, \mathrm{m}), 1.74(1 \mathrm{H}, \mathrm{ddd}, J=12.2,6.1,3.7 \mathrm{~Hz}), 1.64(1 \mathrm{H}, \mathrm{dd}$, $J=8.5,3.4 \mathrm{~Hz}), 1.57-1.51(1 \mathrm{H}, \mathrm{m}), 1.22(3 \mathrm{H}, \mathrm{s}), 0.99(3 \mathrm{H}, \mathrm{d}, J=6.8 \mathrm{~Hz}), 0.96(3 \mathrm{H}, \mathrm{d}, J=6.8 \mathrm{~Hz}), 0.92$ $(3 \mathrm{H}, \mathrm{s}) ;{ }^{13} \mathrm{C} \mathrm{NMR}\left(100 \mathrm{MHz}, \mathrm{CDCl}_{3}\right) \delta 176.1,173.0,141.4,138.2,135.9,118.0,92.9,51.5,48.0$, 40.5, 39.5, 36.7, 32.4, 28.4, 26.5, 25.1, 23.2, 23.0, 21.7, 21.5; IR (neat) $v_{\max } 1678,1658,1616,1248$ $\mathrm{cm}^{-1}$; FAB-MS $[\mathrm{M}]^{+}$calculated for $\mathrm{C}_{20} \mathrm{H}_{28} \mathrm{O}_{3}: 316.2038$, found : 316.2042; $[\alpha]_{\mathrm{D}}{ }^{20.1}+116.3$ (c 0.90 , $\left.\mathrm{CHCl}_{3}\right)$.

\section{(+)-(3aR,5aR,7R)-Methyl}

3,3a,4,5,5a,6,7,8-octahydro-7-(iodomethyl)-1-isopropyl-3a,5a-dimethyl-6-oxo-2H-cyclopenta[a] naphthalene-7-carboxylate (20)<smiles>COC(=O)[C@]1(CI)CC=C2C3=C(C(C)C)CC[C@]3(C)CC[C@]2(C)C1=O</smiles>

$\mathrm{R}_{\mathrm{f}}=0.36$ (hexane/ethyl acetate $=20 / 1$ ); white solid; mp 78.8-81.6 ${ }^{\circ} \mathrm{C} ;{ }^{1} \mathrm{H}$ NMR $\left(400 \mathrm{MHz}, \mathrm{CDCl}_{3}\right) \delta$ $5.50(1 \mathrm{H}, \mathrm{dd}, J=4.4,4.2 \mathrm{~Hz}), 3.81(1 \mathrm{H}, \mathrm{d}, J=9.8 \mathrm{~Hz}), 3.76(3 \mathrm{H}, \mathrm{s}), 3.52(1 \mathrm{H}, \mathrm{d}, J=9.8 \mathrm{~Hz}), 3.25(1 \mathrm{H}$, dd, $J=17.6,4.2 \mathrm{~Hz}), 2.88(1 \mathrm{H}$, sept, $J=6.8 \mathrm{~Hz}), 2.39(1 \mathrm{H}, \mathrm{dd}, J=17.6,4.4 \mathrm{~Hz}), 2.34-2.32(2 \mathrm{H}, \mathrm{m})$, 1.77-1.51 (6H, m), 1.07 (3H, s), $1.02(3 \mathrm{H}, \mathrm{d}, J=6.8 \mathrm{~Hz}), 0.96(3 \mathrm{H}, \mathrm{d}, J=6.8 \mathrm{~Hz}), 0.91(3 \mathrm{H}, \mathrm{s}) ;{ }^{13} \mathrm{C}$ NMR $\left(100 \mathrm{MHz}, \mathrm{CDCl}_{3}\right) \delta 208.2,169.1,143.2,138.0,136.7,118.1,59.4,52.7,49.0,48.0,39.2$, 36.1, 33.6, 32.0, 28.5, 26.5, 23.2, 22.4, 21.6, 21.6, 9.0; IR (KBr) $v_{\max } 1743,1700,562 \mathrm{~cm}^{-1}$; FAB-MS $[\mathrm{M}+\mathrm{H}]^{+}$calculated for $\mathrm{C}_{21} \mathrm{H}_{30} \mathrm{IO}_{3}: 457.1240$, found : 457.1251; $[\alpha]_{\mathrm{D}}^{21.1}+195.3$ (c 1.04, $\left.\mathrm{CHCl}_{3}\right)$. 
(+)-(3aR,5aR,8S(or8R),10Z)-Methyl

2,3,3a,4,5,5a,6,7,8,9-decahydro-1-isopropyl-3a,5a-dimethyl-6-oxocyclohepta[e]indene-8-carbox ylate (21)

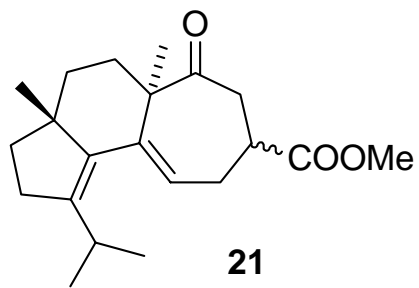

major : $\mathrm{R}_{\mathrm{f}}=0.17$ (hexane/ethyl acetate $\left.=10 / 1\right)$; a colorless oil; ${ }^{1} \mathrm{H}$ NMR $\left(400 \mathrm{MHz}, \mathrm{CDCl}_{3}\right) \delta 5.47(1 \mathrm{H}$, dd, $J=7.7,7.5 \mathrm{~Hz}$ ), $3.73(3 \mathrm{H}, \mathrm{s}), 3.38$ (1H, dd, $J=11.2,9.7 \mathrm{~Hz}), 2.99$ (1H, ddd, $J=18.5,9.7,1.3 \mathrm{~Hz}$ ), $2.83(1 \mathrm{H}$, sept, $J=6.8 \mathrm{~Hz}), 2.71(1 \mathrm{H}, \mathrm{dd}, J=11.2,1.1 \mathrm{~Hz}), 2.38-2.29(4 \mathrm{H}, \mathrm{m}), 1.97(1 \mathrm{H}, \mathrm{ddd}, J=13.0$, 13.0, $5.5 \mathrm{~Hz}), 1.73$ (1H, ddd, $J=12.5,6.6,4.8 \mathrm{~Hz}), 1.69-1.53(3 \mathrm{H}, \mathrm{m}), 1.19$ (1H, ddd, $J=13.6,3.5,3.5$ $\mathrm{Hz}), 1.10(3 \mathrm{H}, \mathrm{s}), 1.02(3 \mathrm{H}, \mathrm{s}), 1.00(3 \mathrm{H}, \mathrm{d}, J=6.8 \mathrm{~Hz}), 0.95(3 \mathrm{H}, \mathrm{d}, J=6.8 \mathrm{~Hz}) ;{ }^{13} \mathrm{C}$ NMR $(100 \mathrm{MHz}$, $\left.\mathrm{CDCl}_{3}\right) \delta 213.7,173.8,143.6,140.6,137.3,120.8,55.2,52.1,48.0,42.2,38.5,37.9,36.4,32.6,28.3$, 26.6, 26.1, 23.6, 21.7, 21.5, 18.5; IR (neat) $v_{\max } 1738,1712 \mathrm{~cm}^{-1}$; FAB-MS $[\mathrm{M}+\mathrm{H}]^{+}$calculated for $\mathrm{C}_{21} \mathrm{H}_{31} \mathrm{O}_{3}: 331.2273$, found : 331.2267; $[\alpha]_{\mathrm{D}}{ }^{26.0}+270.5\left(\mathrm{c} 0.89, \mathrm{CHCl}_{3}\right)$.

minor : $\mathrm{R}_{\mathrm{f}}=0.24$ (hexane/ethyl acetate $=10 / 1$ ); a white solid; $\mathrm{mp} 79.8-82.3^{\circ} \mathrm{C} ;{ }^{1} \mathrm{H}$ NMR (400 MHz, $\left.\mathrm{CDCl}_{3}\right) \delta 5.41(1 \mathrm{H}, \mathrm{dd}, J=7.9,7.2 \mathrm{~Hz}), 3.71(3 \mathrm{H}, \mathrm{s}), 3.47(1 \mathrm{H}, \mathrm{dd}, J=10.8,10.8 \mathrm{~Hz}), 3.07(1 \mathrm{H}, \mathrm{dddd}$, $J=10.8,6.8,6.8,1.8 \mathrm{~Hz}), 2.81(1 \mathrm{H}, \mathrm{sept}, J=6.8 \mathrm{~Hz}), 2.50-2.42(2 \mathrm{H}, \mathrm{m}), 2.38-2.22(3 \mathrm{H}, \mathrm{m}), 2.05(1 \mathrm{H}$, ddd, $J=13.2,13.2,5.3 \mathrm{~Hz}), 1.74(1 \mathrm{H}, \mathrm{ddd}, J=12.5,6.6,4.8 \mathrm{~Hz}), 1.66-1.58(3 \mathrm{H}, \mathrm{m}), 1.16(1 \mathrm{H}$, ddd, $J=13.6,3.5,3.5 \mathrm{~Hz}), 1.10(3 \mathrm{H}, \mathrm{s}), 1.05(3 \mathrm{H}, \mathrm{s}), 0.99(1 \mathrm{H}, \mathrm{d}, J=6.8 \mathrm{~Hz}), 0.93(1 \mathrm{H}, \mathrm{d}, J=6.8 \mathrm{~Hz}) ;{ }^{13} \mathrm{C}$ NMR $\left(100 \mathrm{MHz}, \mathrm{CDCl}_{3}\right) \delta 214.3,173.9,143.7,140.3,137.2,119.7,55.4,52.1,48.0,42.4,38.4$, $38.1,36.5,32.7,28.3,26.6,26.0,23.6,21.6,21.6,18.5$; IR (KBr) $v_{\max } 1745,1703 \mathrm{~cm}^{-1}$; FAB-MS $[\mathrm{M}+\mathrm{H}]^{+}$calculated for $\mathrm{C}_{21} \mathrm{H}_{31} \mathrm{O}_{3}: 331.2273$, found : 331.2267; $[\alpha]_{\mathrm{D}}^{24.9}+241.1\left(\mathrm{c} 1.06, \mathrm{CHCl}_{3}\right)$.

\section{(+)-(3aR,5aR,8E,10Z)-Methyl}

\section{2,3,3a,4,5,5a,6,7-octahydro-1-isopropyl-3a,5a-dimethyl-6-oxocyclohepta[e]indene-8-carboxylat} e (2)

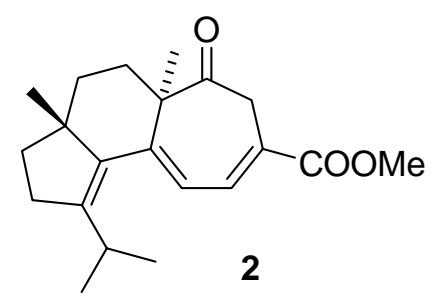

$\mathrm{R}_{\mathrm{f}}=0.24$ (hexane/ethyl acetate=10/1); a pale yellow oil; ${ }^{1} \mathrm{H}$ NMR $\left(400 \mathrm{MHz}, \mathrm{CDCl}_{3}\right) \delta 7.27(1 \mathrm{H}$, dd, $J=6.1,2.0 \mathrm{~Hz}), 5.95(1 \mathrm{H}, \mathrm{d}, J=6.1 \mathrm{~Hz}), 3.86(1 \mathrm{H}, \mathrm{dd}, J=11.0,0.5 \mathrm{~Hz}), 3.79(3 \mathrm{H}, \mathrm{s}), 3.39(1 \mathrm{H}, \mathrm{ddd}$, $J=11.0,2.0,1.0 \mathrm{~Hz}), 2.87(1 \mathrm{H}$, sept, $J=6.8 \mathrm{~Hz}), 2.40(2 \mathrm{H}, \mathrm{dd}, J=9.0,5.6 \mathrm{~Hz}), 2.02(1 \mathrm{H}, \mathrm{ddd}, J=13.0$, 13.0, $4.2 \mathrm{~Hz}), 1.80-1.55(4 \mathrm{H}, \mathrm{m}), 1.44(1 \mathrm{H}, \mathrm{ddd}, J=13.4,4.2,4.2 \mathrm{~Hz}), 1.17(3 \mathrm{H}, \mathrm{s}), 1.05(3 \mathrm{H}, \mathrm{d}$, 
$J=6.8 \mathrm{~Hz}), 1.02(3 \mathrm{H}, \mathrm{s}), 1.00(3 \mathrm{H}, \mathrm{d}, J=6.8 \mathrm{~Hz}) ;{ }^{13} \mathrm{C} \mathrm{NMR}\left(100 \mathrm{MHz}, \mathrm{CDCl}_{3}\right) \delta 205.2,166.3,148.7$, 144.2, 139.6, 135.8, 123.7, 121.9, 56.9, 52.5, 49.3, 40.8, 38.6, 36.0, 34.2, 28.6, 26.8, 23.8, 22.4, 21.6, 21.6; IR (neat) $v_{\max } 1716,1590 \mathrm{~cm}^{-1}$; FAB-MS $[\mathrm{M}+\mathrm{H}]^{+}$calculated for $\mathrm{C}_{21} \mathrm{H}_{29} \mathrm{O}_{3}: 329.2117$, found : 329.2113; $[\alpha]^{26.1}+53.1\left(\mathrm{c} 0.92, \mathrm{CHCl}_{3}\right)$.

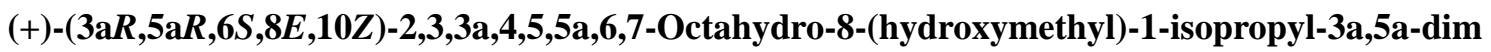
ethylcyclohepta[e]inden-6-ol

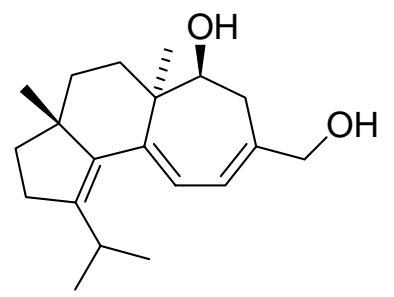

$\mathrm{R}_{\mathrm{f}}=0.14$ (hexane/ethyl acetate $\left.=2 / 1\right)$; a colorless oil; ${ }^{1} \mathrm{H}$ NMR $\left(400 \mathrm{MHz}, \mathrm{CDCl}_{3}\right) \delta 6.04(1 \mathrm{H}, \mathrm{m})$, $5.60(1 \mathrm{H}, \mathrm{d}, J=8.3 \mathrm{~Hz}), 4.11(1 \mathrm{H}, \mathrm{s}), 3.59(1 \mathrm{H}, \mathrm{m}), 2.83(1 \mathrm{H}$, sept, $J=6.8 \mathrm{~Hz}), 2.73(1 \mathrm{H}, \mathrm{d}, J=17.6$ Hz), 2.59 (1H, dd, $J=17.6,5.9 \mathrm{~Hz}), 2.46(1 \mathrm{H}, \mathrm{ddd}, J=13.9,9.3,9.3 \mathrm{~Hz}), 2.37-2.32(2 \mathrm{H}, \mathrm{m}), 1.74-1.54$ $(4 \mathrm{H}, \mathrm{m}), 1.24(1 \mathrm{H}, \mathrm{ddd}, J=12.6,3.5,3.5 \mathrm{~Hz}), 1.00(3 \mathrm{H}, \mathrm{d}, J=6.8 \mathrm{~Hz}), 0.95(3 \mathrm{H}, \mathrm{s}), 0.94(3 \mathrm{H}, \mathrm{d}, J=6.8$ $\mathrm{Hz}), 0.93(3 \mathrm{H}, \mathrm{s}) ;{ }^{13} \mathrm{C}$ NMR $\left(100 \mathrm{MHz}, \mathrm{CDCl}_{3}\right) \delta 143.8,143.2,141.9,138.0,121.7,119.3,74.6$, $68.8,48.3,47.2,38.6,36.8,34.1,32.9,28.6,26.9,26.2,23.8,21.6,21.6$; IR (neat) $v_{\max } 3364,1596$ $\mathrm{cm}^{-1}$; FAB-MS $[\mathrm{M}]^{+}$calculated for $\mathrm{C}_{20} \mathrm{H}_{30} \mathrm{O}_{2}: 302.2246$, found : 302.2248; $[\alpha]_{\mathrm{D}}{ }^{28.8}+110.0(\mathrm{c} 0.95$, $\left.\mathrm{CHCl}_{3}\right)$.

(+)-(3aR,5aR,6S,8E,10Z)-2,3,3a,4,5,5a,6,7-Octahydro-6-hydroxy-1-isopropyl-3a,5a-dimethylcyc lohepta[e]indene-8-carbaldehyde (allocyathin $\left.B_{2}\right)(1)$

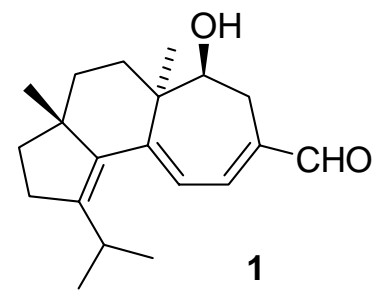

$\mathrm{R}_{\mathrm{f}}=0.28$ (hexane/ethyl acetate $\left.=4 / 1\right)$; a pale yellow oil; ${ }^{1} \mathrm{H}$ NMR $\left(400 \mathrm{MHz}, \mathrm{CDCl}_{3}\right) \delta 9.45(1 \mathrm{H}, \mathrm{s})$, $6.81(1 \mathrm{H}, \mathrm{dd}, J=8.1,2.4 \mathrm{~Hz}), 5.94(1 \mathrm{H}, \mathrm{d}, J=8.1 \mathrm{~Hz}), 3.72(1 \mathrm{H}, \mathrm{dd}, \mathrm{J}=8.5,6.1), 3.16(1 \mathrm{H}, \mathrm{dd}, J=18.3$, $5.9 \mathrm{~Hz}), 2.83(1 \mathrm{H}$, sept, $J=6.8 \mathrm{~Hz}), 2.55(1 \mathrm{H}, \mathrm{d}, J=18.6 \mathrm{~Hz}), 2.57-2.48(1 \mathrm{H}, \mathrm{m}), 2.45-2.37(2 \mathrm{H}, \mathrm{m})$, 1.77-1.63 (4H, m), $1.34(1 \mathrm{H}$, ddd, $J=13.9,3.7,3.4 \mathrm{~Hz}), 1.05(3 \mathrm{H}, \mathrm{d}, J=6.8 \mathrm{~Hz}), 1.00(3 \mathrm{H}, \mathrm{s}), 0.97$ $(3 \mathrm{H}, \mathrm{d}, J=6.8 \mathrm{~Hz}), 0.96(3 \mathrm{H}, \mathrm{s}) ;{ }^{13} \mathrm{C} \mathrm{NMR}\left(100 \mathrm{MHz}, \mathrm{CDCl}_{3}\right) \delta 194.2,155.1,146.2,144.3,141.9$, $137.9,119.3,74.2,49.1,48.3,38.5,36.5,33.8,29.2,29.0,26.9,26.4,23.8,21.6,21.6$; IR (neat) $v_{\max }$ $3586,1684,1570 \mathrm{~cm}^{-1}$; FAB-MS $[\mathrm{M}]^{+}$calculated for $\mathrm{C}_{20} \mathrm{H}_{28} \mathrm{O}_{2}: 300.2089$, found : 300.2086 ; $[\alpha]_{\mathrm{D}}{ }^{29.6}$ $+85.3\left(\mathrm{c} 0.97, \mathrm{CH}_{3} \mathrm{OH}\right)$. 
ORTEP Drawing of $\mathbf{1 5}$

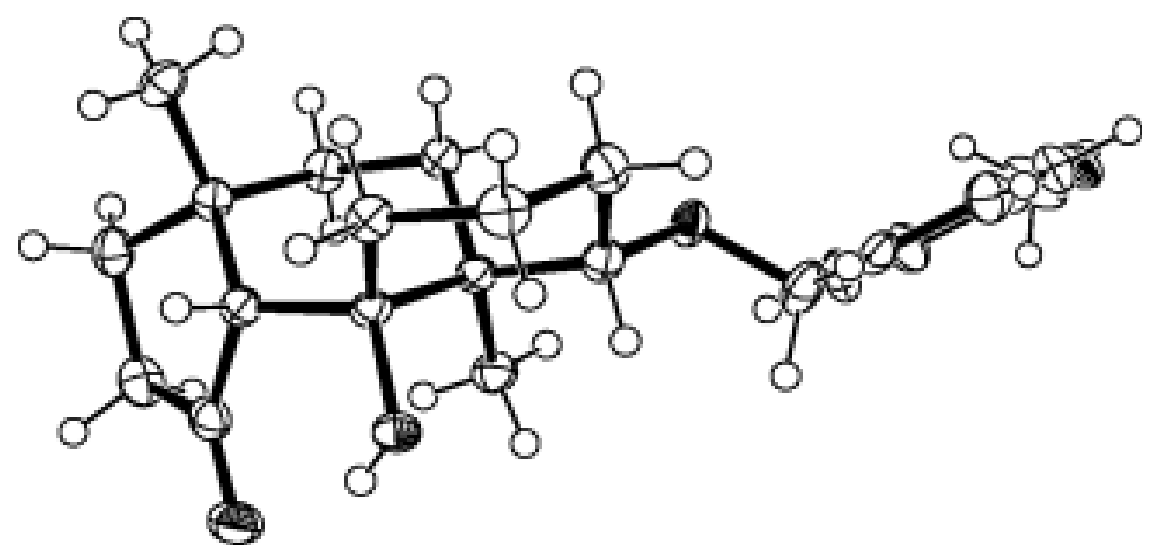

